\title{
Iterative Equalization and TCM Decoding with Refined Channel Value*
}

\author{
Pei Xiao, Rolando Carrasco \\ School of Electrical, Electronic and Computer Engineering \\ University of Newcastle Upon Tyne NE1 7RU, United Kingdom \\ E-mail: pei.xiao, r.carrasco@ncl.ac.uk \\ Ian Wassell \\ Digital Technology Group, Computer Laboratory, University of Cambridge \\ 15 JJ Thomson Avenue, CB3 0FD, United Kingdom \\ E-mail: i jw24@eng.cam.ac.uk
}

\begin{abstract}
We present a new method for the iterative equalization and decoding of multilevel trellis coded modulation (TCM) signals over frequency selective channels. Results show that the proposed algorithm achieves better performance compared to the previous work on the MMSE filter-based turbo equalization for a non-binary coded modulation scheme. The performance gain is accomplished by utilizing the combined modulation and coding nature of TCM and passing the refined signal obtained from different paths to the TCM decoder as the channel value in addition to the a priori probabilities.
\end{abstract}

Keywords: turbo equalization, TCM, intersymbol interference.

\section{INTRODUCTION AND PROBLEM FORMULATION}

In a mobile communications environment, multipath propagation causes dispersion of transmitted signals. The time delay spread causes intersymbol interference (ISI) and degrades system performance. Therefore, equalization methods which can mitigate the effects of ISI must be employed. A frequency selective channel can be described as a rate one convolutional code defined over the field of real or complex numbers, so the combination of a channel code and ISI channel can be viewed as a serial concatenation system, and can be decoded using turbo processing principle. Douillard, et al. proposed turbo equalization in [1] which combines equalization with channel decoding to remove the effect of intersymbol interference caused by frequency selective fading channels. The receiver consists of two trellis-based detectors, one for equalization and one for decoding. It is shown that turbo equalization significantly improves the performance over separate equalization and decoding. However, for channels with large delay spreads and for large constellation sizes, the trellis based turbo equalization scheme suffers from prohibitive computational complexity. In [2], [3], Wang, Tuchler, et al, introduced new approaches to combining equalization based on the linear minimum mean square error (MMSE) filtering with decoding. It was shown that this new approach provides a tremendous

\footnotetext{
*This work has been presented in part at the IEEE Global Telecommunications Conference (Globecom) 2005.
}

complexity reduction with acceptable performance degradation compared to the trellis-based approach.

Trellis Coded Modulation (TCM) is a powerful technique to improve bandwidth efficiency without increasing the transmitted power. It combines modulation and coding by optimizing the Euclidean distance between codewords. Turbo techniques were applied to the trellis-based equalization of TCM signals in [4], [5]. Despite its good performance, the major disadvantage of this approach is the exponentially increasing complexity of the equalizer which is significant for channels with long impulse response or large signal constellation size. To reduce the complexity, the MMSE filter-based turbo equalization originally proposed in [2], [3] was extended to the TCM signals in [6], [7]. Although it reduces the complexity from exponential to cubic compared to the trellis-based approach, this algorithm is still computationally intensive due to matrix inversion required at each symbol period.

In this correspondence, we obtain symbol a posteriori probabilities directly from the output of the interference canceler, thus the MMSE filtering and its inherent matrix inversion procedure are avoided. In order to improve the system performance, we modify the TCM decoder such that it takes the refined channel value in addition to symbol a posteriori probabilities as inputs and produces both symbol extrinsic probabilities and log-likelihood ratio (LLR) values for information bits at the output. This is in contrast to previous work on the filter-based approach in which the TCM decoder only takes symbol a posteriori probabilities as its input. A comparison between the proposed scheme and MMSE filter-based equalization is provided in this correspondence to demonstrate the effectiveness of the proposed algorithm.

The transmission system under study is shown in Figure 1. The information sequence $\left\{b_{n}\right\}$ is TCM encoded and mapped into M-PSK or M-QAM symbols $\left\{s_{n}\right\}$. The coded and modulated symbol sequence is block interleaved into $\left\{s_{n}^{\prime}\right\}$ and transmitted over the ISI channel, which can be modeled by an equivalent baseband system where the concatenation of the transmit filter, the channel and the receive filter, is represented by a discrete-time $L$-tap transversal filter with finite-length impulse response $h_{n}=\sum_{l=0}^{L-1} h_{l} \delta_{n-l}$ where the complex channel coefficients $h_{l}$ are assumed time invariant during the 


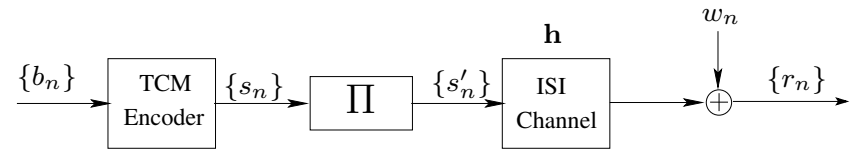

Fig. 1. Block diagram of the transmission scheme.

transmission of one block of data. The transmit filter and receive filter are chosen such that their composite frequency response fulfills Nyquist criterion and the receive filter is matched to the transmit filter [8]. With symbol rate sampling at the receiver, the received samples $\left\{r_{n}\right\}$ can be expressed as

$$
r_{n}=\sum_{l=0}^{L-1} s_{n-l}^{\prime} h_{l}+w_{n},
$$

where $s_{n}^{\prime}$ is the coded and interleaved symbol, and $w_{n}$ is the complex additive white Gaussian noise with zero mean and variance $N_{0}$. In this work, we assume that the multipaths are resolvable and signals experience frequency selective fading. In case where the sample period is much shorter than channel tap spacing, the discrete time channel model can be modified accordingly, e.g., zeros can be inserted between adjacent channel taps (the number of zeros inserted depends on the ratio between the sample period and the channel tap spacing).

\section{Iterative Equalization AND TCM Decoding}

The block diagram of the proposed equalization and TCM decoding scheme is shown in Figure 2. In the first pass of the turbo equalization operation, the channel is estimated using a training sequence. At this stage, we also derive an initial estimate (hard decision) of the transmitted symbols using, e.g., a zero-forcing (ZF) equalizer or a decision feedback equalizer (DFE). With channel estimates, the ZF equalizer can be easily designed by inverting the channel transfer function. However, it leads to noise enhancement, especially at low SNR. Therefore, we choose DFE for the initial symbol estimate. The equalizer coefficients are obtained adaptively using the recursive least square (RLS) algorithm [8].

With symbol and channel estimates, we perform soft interference cancellation and pass the refined signal to the TCM decoder via a maximum ratio combiner (MRC). In the meantime, the a posteriori symbol probabilities at each time instant $n$ are collected in an M-ary vector and passed to the decoder as a priori information. The TCM decoder computes the extrinsic probability of the TCM symbols as well as the log-likelihood ratio (LLR) of the information bits. The former is interleaved and sent back to the equalizer as a priori information to start the subsequent iteration. The latter is used to make hard decision on the transmitted information bits. In this way, the equalization and TCM decoding are performed jointly in an iterative manner. The equalization and TCM decoding blocks will now be described in detail.

\section{A. Equalization Algorithm}

As shown in Figure 2, the proposed equalizer includes a soft interference canceler (SIC) followed by i) a mapper to compute a posteriori probabilities; ii) an MRC to obtain refined channel values $\left(y_{n}^{\prime}\right.$ and its deinterleaved version $\left.y_{n}\right)$ based on $\tilde{r}_{n}$, the interference canceled version of the received signal $r_{n}$. Note that $y_{n}$ is the refined channel value, and $r_{n}$ is the original channel value. This algorithm is different from those presented in [2], [3], which are based on the computation of LLRs. Here, modifications have to made to accommodate multilevel modulation [9]. In particular, one has to compute extrinsic probabilities rather than LLRs in order to allow the application of turbo equalization to TCM systems.

Based on (1), the ISI canceled signals after performing interference cancellation using soft estimates of the transmitted symbols become

$$
\begin{aligned}
\tilde{r}_{n} & =h_{0} s_{n}^{\prime}+\left(h_{1} s_{n-1}^{\prime}-\hat{h}_{1} \bar{s}_{n-1}^{\prime}\right)+\ldots+w_{n} \\
\tilde{r}_{n+1} & =h_{1} s_{n}^{\prime}+\left(h_{0} s_{n+1}^{\prime}-\hat{h}_{0} \bar{s}_{n+1}^{\prime}\right)+\ldots+w_{n+1} \\
\vdots & \\
\tilde{r}_{n+L-1} & =h_{L-1} s_{n}^{\prime}+\left(h_{0} s_{n+L-1}^{\prime}-\hat{h}_{0} \bar{s}_{n+L-1}^{\prime}\right)+\ldots+w_{n+L-1},
\end{aligned}
$$

where $\tilde{r}_{n+i}$ denotes the ISI canceled version of the received signal $r_{n+i}$ after canceling the contributions from all the symbols other than $s_{n}$, which is the symbol of interest. $\hat{h}_{l}$ is the estimate of the channel coefficient $h_{l}$ and $\bar{s}_{n+i}^{\prime}$ denotes the soft estimate of $s_{n+i}^{\prime}$, which is computed as

$$
\bar{s}_{n+i}^{\prime}=\mathrm{E}\left[s_{n+i}^{\prime}\right]=\sum_{q=1}^{M} P\left(s_{n+i}^{\prime}=s_{q}\right) s_{q},
$$

where $P\left(s_{n+i}^{\prime}=s_{q}\right)$ is the a priori probability of the symbol $s_{n+i}^{\prime}$, which is initialized by the DFE at the first stage and provided by the TCM Log-MAP decoder at the following stages. The above formulas can be written in vector form as

$$
\tilde{\mathbf{r}}_{n}=\left[\begin{array}{c}
\tilde{r}_{n} \\
\tilde{r}_{n+1} \\
\vdots \\
\tilde{r}_{n+L-1}
\end{array}\right]=\left[\begin{array}{c}
h_{0} \\
h_{1} \\
\vdots \\
h_{L-1}
\end{array}\right] s_{n}^{\prime}+\left[\begin{array}{c}
v_{n} \\
v_{n+1} \\
\vdots \\
v_{n+L-1}
\end{array}\right]=\mathbf{h} s_{n}^{\prime}+\mathbf{v}
$$

where $\mathbf{h}=\left[h_{0}, \ldots, h_{L-1}\right]^{T}$ denotes the channel vector, and $\mathbf{v}=\left[v_{n}, \ldots, v_{n+L-1}\right]^{T}$ stands for the vector of combined noise and interference cancellation residual. This interference cancellation procedure is similar to the one proposed in [7] where the symbol a posteriori probabilities are derived from the output of an MMSE filter. Refer to [7] for a detailed description of this MMSE filter-based algorithm. Next, we shall introduce a different approach to compute the symbol a posteriori probabilities without using an MMSE filter.

Each element of $\mathbf{v}$ can be approximated as a complex Gaussian random variable with zero mean and variance $N_{v}$ [10]. This approximation simplifies the derivation although it does not rigorously hold. In our case, the assumption of zero mean holds if i) $\mathrm{E}\left[s_{n+i}^{\prime}\right]=\bar{s}_{n+i}^{\prime}$; ii) the channel estimation is unbiased, i.e., $\mathrm{E}\left[\hat{h}_{l}\right]=h_{l}$. The first condition is fulfilled according to (2). In this work, we employ a modified maximum likelihood channel estimator introduced in [11], which has been shown to be unbiased. Since the transmitted data symbols 


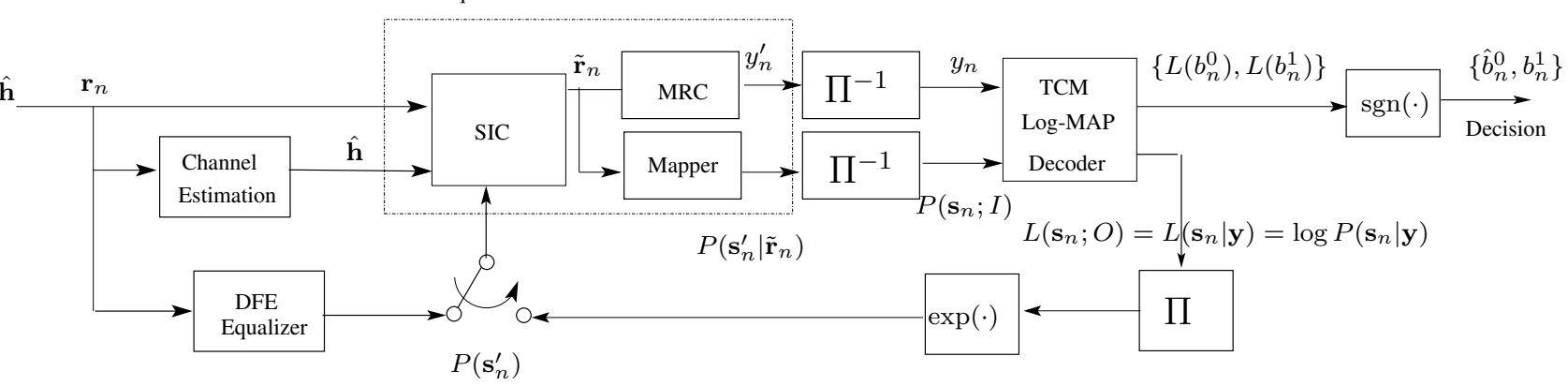

Fig. 2. Block diagram for the proposed iterative equalization and TCM decoding scheme.

$\left\{s_{n}^{\prime}\right\}$ have unitary variance, it can be derived from (3) that $\mathrm{E}\left[\left\|\tilde{\mathbf{r}}_{n}\right\|^{2}\right]=\mathrm{E}\left[\left(\mathbf{h} s_{n}^{\prime}+\mathbf{v}\right)\left(\mathbf{h} s_{n}^{\prime}+\mathbf{v}\right)^{*}\right]=\mathrm{E}\left[\left\|\mathbf{h} s_{n}^{\prime}\right\|^{2}\right]+\mathrm{E}\left[\|\mathbf{v}\|^{2}\right]$

$$
=\mathrm{E}\left[\|\mathbf{h}\|^{2}\right]+L N_{v}=\mathcal{P}+L N_{v}
$$

where $\mathcal{P}=\sum_{l=0}^{L-1}\left|h_{l}\right|^{2}$ denotes the total received power from all the paths. The variance of noise plus residual interference can be derived statistically as

$$
N_{v}=\frac{\mathrm{E}\left[\left\|\tilde{\mathbf{r}}_{n}\right\|^{2}\right]-\mathcal{P}}{L} \approx \frac{\left\|\overline{\tilde{\mathbf{r}}_{n}}\right\|^{2}-\mathcal{P}}{L}
$$

where $\left\|\overline{\tilde{\mathbf{r}}_{n}}\right\|^{2}$ is the energy of the vector $\tilde{\mathbf{r}}_{n}$ averaged over the whole block of symbols. In case of perfect cancellation, each element of $\mathbf{v}$ only contains the noise, i.e., $v_{n+i}=w_{n+i}$, and $N_{v}=N_{0}$.

The conditional probability density function (PDF) of $\tilde{\mathbf{r}}_{n}$ can now be represented by

$$
\begin{aligned}
& p\left(\tilde{\mathbf{r}}_{n} \mid s_{n}^{\prime}=s_{i}\right) \approx \frac{1}{\left(\pi N_{v}\right)^{L}} \exp \left(-\frac{\left\|\tilde{\mathbf{r}}_{n}-\mathbf{h} s_{i}\right\|^{2}}{N_{v}}\right) \\
& =\frac{1}{\left(\pi N_{v}\right)^{L}} \exp \left(-\frac{\left\|\tilde{\mathbf{r}}_{n}\right\|^{2}+\left\|\mathbf{h} s_{i}\right\|^{2}-2 \operatorname{Re}\left\{\left(\mathbf{h} s_{i}\right)^{*} \tilde{\mathbf{r}}_{n}\right\}}{N_{v}}\right),
\end{aligned}
$$

where the superscript operator ()$^{*}$ is the conjugate transpose operation when applied to matrices and vectors, and simply the conjugate when applied to scalars. We know that

$$
\begin{aligned}
& P\left(s_{n}^{\prime}=s_{i} \mid \tilde{\mathbf{r}}_{n}\right)=\frac{p\left(\tilde{\mathbf{r}}_{n} \mid s_{n}^{\prime}=s_{i}\right) P\left(s_{n}^{\prime}=s_{i}\right)}{p\left(\tilde{\mathbf{r}}_{n}\right)} ; \\
& p\left(\tilde{\mathbf{r}}_{n}\right)=\sum_{q=1}^{M} P\left(s_{n}^{\prime}=s_{q}\right) p\left(\tilde{\mathbf{r}}_{n} \mid s_{n}^{\prime}=s_{q}\right) .
\end{aligned}
$$

In a PSK constellation, all the signals lie on the unit circle, therefore have the same energy, i.e., $E_{s}=\left|s_{1}\right|^{2}=$ $\ldots=\left|s_{M}\right|^{2}$, and $\left\|\tilde{\mathbf{r}}_{n}\right\|^{2}$ in (5) is the same for all the signal alternatives. Combining (5) and (6) yields

$P\left(s_{n}^{\prime}=s_{i} \mid \tilde{\mathbf{r}}_{n}\right)=\frac{P\left(s_{n}^{\prime}=s_{i}\right) \exp \left(2 \operatorname{Re}\left\{\left(\mathbf{h} s_{i}\right)^{*} \tilde{\mathbf{r}}_{n}\right\} / N_{v}\right)}{\sum_{q=1}^{M} P\left(s_{n}^{\prime}=s_{q}\right) \exp \left(2 \operatorname{Re}\left\{\left(\mathbf{h} s_{q}\right)^{*} \tilde{\mathbf{r}}_{n}\right\} / N_{v}\right)}$

$=\frac{e^{\frac{2 \operatorname{Re}\left\{\left(\mathbf{h} s_{i}\right)^{*} \tilde{\mathbf{r}}_{n}\right\}}{N_{v}}+\log P\left(s_{n}^{\prime}=s_{i}\right)}}{e^{\max *\left[\frac{2 \operatorname{Re}\left\{\left(\mathbf{h} s_{1}\right)^{*} \tilde{\mathbf{r}}_{n}\right\}}{N_{v}}+\log P\left(s_{n}^{\prime}=s_{1}\right), \cdots, \frac{2 \operatorname{Re}\left\{\left(\mathbf{h} s_{M}\right)^{*} \tilde{\mathbf{r}}_{n}\right\}}{N_{v}}+\log P\left(s_{n}^{\prime}\right.\right.}}$

where the function $\max ^{*}[]$ is defined as $\max ^{*}[x, y]=$ $\ln \left(e^{x}+e^{y}\right)=\max [x, y]+\ln \left(1+e^{-|x-y|}\right)$, i.e., the $\max$ operation compensated with a correction term $\ln \left(1+e^{-|x-y|}\right)$. Also $\max ^{*}[x, y, z]=\max ^{*}\left[\max ^{*}[x, y], z\right]$, etc.. The symbols are assumed to be equally probable at the first iteration, i.e., $P\left(s_{n}^{\prime}=s_{1}\right)=P\left(s_{n}^{\prime}=s_{2}\right)=\ldots=P\left(s_{n}^{\prime}=s_{M}\right)=1 / M$. The original channel vector $\mathbf{h}$ in (8) is unknown, and has to be replaced by its estimate $\hat{\mathbf{h}}$. Direct implementation of (7) leads to numerical instability at high SNR, which can be tackled by performing the calculation in the log domain as shown in (8), similar to the idea presented in [12].

Note that (7) and (8) only apply to PSK signals. For QAM signals, each signal alternative might not have the same energy. Therefore, equation (7) should be modified as

$P\left(s_{n}^{\prime}=s_{i} \mid \tilde{\mathbf{r}}_{n}\right)=\frac{P\left(s_{n}^{\prime}=s_{i}\right) \exp \left(\frac{2 \operatorname{Re}\left\{\left(\mathbf{h} s_{i}\right)^{*} \tilde{\mathbf{r}}_{n}\right\}-\left\|\mathbf{h} s_{i}\right\|^{2}}{N_{v}}\right)}{\sum_{q=1}^{M} P\left(s_{n}^{\prime}=s_{q}\right) \exp \left(\frac{2 \operatorname{Re}\left\{\left(\mathbf{h} s_{q}\right)^{*} \tilde{\mathbf{r}}_{n}\right\}-\left\|\mathbf{h} s_{q}\right\|^{2}}{N_{v}}\right)}$,

and its Log-MAP implementation can be obtained similarly.

We form the vectors $P\left(\mathbf{s}_{n}^{\prime}\right)$ and $P\left(\mathbf{s}_{n}^{\prime} \mid \tilde{\mathbf{r}}_{n}\right)$ as

$$
\begin{aligned}
P\left(\mathbf{s}_{n}^{\prime}\right) & =\left[\begin{array}{llll}
P\left(s_{n}^{\prime}=s_{1}\right) & P\left(s_{n}^{\prime}=s_{2}\right) & \ldots & P\left(s_{n}^{\prime}=s_{M}\right)
\end{array}\right]^{T} ; \\
P\left(\mathbf{s}_{n}^{\prime} \mid \tilde{\mathbf{r}}_{n}\right) & =\left[\begin{array}{llll}
P\left(s_{n}^{\prime}=s_{1} \mid \tilde{\mathbf{r}}_{n}\right) & \ldots & P\left(s_{n}^{\prime}=s_{M} \mid \tilde{\mathbf{r}}_{n}\right)
\end{array}\right]^{T} .
\end{aligned}
$$

The former is the a priori probabilities vector of the symbol $s_{n}^{\prime}$. It is an input to the SIC block. The latter is the output of the mapper. Each element of $P\left(\mathbf{s}_{n}^{\prime}\right)$ is initialized as

$$
P\left(s_{n}^{\prime}=s_{i}\right)= \begin{cases}1 & \text { if } \hat{s}_{n}^{\prime}=s_{i}, \\ 0 & \text { otherwise }\end{cases}
$$

where $\hat{s}_{n}^{\prime}$ is the hard decision from the DFE equalizer. At the following stages, this a priori probability vector is formed by the extrinsic information provided by the decoding step, i.e., $P\left(\mathbf{s}_{n}^{\prime}\right)=\Pi\left\{P\left(\mathbf{s}_{n} ; O\right)\right\}$, where $P\left(\mathbf{s}_{n} ; O\right)=\exp \left[L\left(\mathbf{s}_{n} ; O\right)\right]$ is the output of the TCM Log-MAP decoder. We use the notations $P\left(\mathbf{s}_{n} ; I\right), P\left(\mathbf{s}_{n} ; O\right)$ to denote the input and output of the TCM decoder.

The refined signal to be passed to the TCM decoder as the channel value is obtained based on the interference canceled signal vector $\tilde{\mathbf{r}}_{n}$ using maximum ratio combining, i.e.,

$$
y_{n}^{\prime}=\hat{\mathbf{h}}^{*} \tilde{\mathbf{r}}_{n}=\hat{\mathbf{h}}^{*} \mathbf{h} s_{n}^{\prime}+\hat{\mathbf{h}}^{*} \mathbf{v}=\hat{\mathbf{h}}^{*} \mathbf{h} s_{n}^{\prime}+\eta^{\prime},
$$

where $\hat{\mathbf{h}}$ is an estimate of $\mathbf{h}, \eta^{\prime}$ is zero mean Gaussian random variable with variance $N_{\eta}=\mathcal{P} N_{v}$.

In contrast to the MMSE filter-based approach introduced in [6], [7], the derivation of symbol a posteriori probabilities 
described previously does not involve matrix inversion. The presented algorithm reduces the computational complexity from $O\left(L^{3}\right)$ to $O\left(L^{2}\right)$, where $L$ is the number of channel taps. A quantitative complexity comparison is given in Table I, which shows the required number of complex multiplications/divisions, additions/subtractions, etc. for each TCM symbol estimate by the two different schemes. The figures for the MMSE scheme are based on its Log-MAP implementation.

\section{B. TCM Decoding Algorithm}

The proposed TCM Log-MAP decoder takes the deinterleaved probability vector $P\left(\mathbf{s}_{n} ; I\right)=\Pi^{-1}\left\{P\left(\mathbf{s}_{n}^{\prime} \mid \tilde{\mathbf{r}}_{n}\right)\right\}$ as priori information as well as the combined signal $y_{n}=$ $\Pi^{-1}\left(y_{n}^{\prime}\right)$ as channel input. It outputs the log-likelihood ratio of the information bits $\left\{L\left(b_{n}^{0}\right), L\left(b_{n}^{1}\right)\right\}$ and the refined probability vector $P\left(\mathbf{s}_{n} ; O\right)=P\left(\mathbf{s}_{n} \mid \mathbf{y}\right)$ based on the code constraints and the trellis structure of the TCM code where $\mathbf{y}=\left[\begin{array}{llll}y_{1} & y_{2} & \ldots & y_{L_{b}}\end{array}\right]$ and $L_{b}$ denotes the symbol block length. Based on (9), each element of $y$ is defined as

$$
\begin{aligned}
y_{n} & =\Pi^{-1}\left(y_{n}^{\prime}\right)=\Pi^{-1}\left(\hat{\mathbf{h}}^{*} \mathbf{h} s_{n}^{\prime}+\eta^{\prime}\right)=\hat{\mathbf{h}}^{*} \mathbf{h} s_{n}+\eta \\
& \approx\|\mathbf{h}\|^{2} s_{n}+\eta=\mathcal{P} s_{n}+\eta,
\end{aligned}
$$

where $\eta$ is zero mean Gaussian random variable with variance $N_{\eta}=\mathcal{P} N_{v}$ (interleaving does not change the noise PDF). As shown in (9), the input noise is filtered by the channel which makes the successive noise samples $\eta^{\prime}$ correlated. However, due to the deinterleaving operation, the deinterleaved noise $\eta$ can be approximated as white Gaussian noise. This approximation will be used later on in the derivation of branch metrics for the TCM decoder. The LLR values $\left\{L\left(b_{n}^{0}\right), L\left(b_{n}^{1}\right)\right\}$ are used for making hard decision on the transmitted information bits; while the logarithm of extrinsic probability $L\left(\mathbf{s}_{n} ; O\right)=$ $L\left(\mathbf{s}_{n} \mid \mathbf{y}\right)=\log P\left(\mathbf{s}_{n} \mid \mathbf{y}\right)$ is fed back to equalizer as the a priori probability vector for the next iteration.

The MAP algorithm for non-binary TCM decoder was derived in [13]. In the following, we briefly present the TCM Log-MAP decoding algorithm. Since the issue of TCM decoding with channel value is not addressed in the previous literature, our emphasis will be placed on how the refined channel value (in addition to symbol a priori information) is used in the computation of the branch metrics. Let us denote $\alpha_{n}\left(\sigma^{\prime}\right)$ as the state metric at the $n^{t h}$ trellis node, and $\beta_{n+1}(\sigma)$ as the state metric for the portion of the trellis beyond the $n^{\text {th }}$ trellis node, where $\sigma^{\prime}$ and $\sigma$ are the generic states at the $n^{t h}$ and $(n+1)^{t h}$ nodes, respectively. The logarithm of $P\left(s_{n}=s_{i} \mid \mathbf{y}\right)$ is computed as

$$
\begin{aligned}
& L\left(s_{n}=s_{i} \mid \mathbf{y}\right)=\log P\left(s_{n}=s_{i} \mid \mathbf{y}\right) \\
& =\max _{\sigma^{\prime}, \sigma: s_{i}} *\left[\alpha_{n}\left(\sigma^{\prime}\right)+\gamma_{n}^{*}\left(\sigma^{\prime}, \sigma\right)+\beta_{n+1}(\sigma)\right] \\
& \quad-\max _{\sigma^{\prime}, \sigma}^{*}\left[\alpha_{n}\left(\sigma^{\prime}\right)+\gamma_{n}\left(\sigma^{\prime}, \sigma\right)+\beta_{n+1}(\sigma)\right],
\end{aligned}
$$

where $\gamma_{n}\left(\sigma^{\prime}, \sigma\right), \gamma_{n}^{*}\left(\sigma^{\prime}, \sigma\right)$ are the metrics for the branch connecting state $\sigma^{\prime}$ at node $n$ and $\sigma$ at node $(n+1)$. Expressions defining these terms will be given later. The notation $\sigma^{\prime}, \sigma: s_{i}$ denotes the sets of state pairs $\left(\sigma^{\prime}, \sigma\right)$ in the TCM trellis corresponding to symbol $s_{i}$, and $\sigma^{\prime}, \sigma$ denotes all the possible sets of state pairs where the transition $\left(\sigma^{\prime}, \sigma\right)$ is possible.
We modify the TCM decoder in order to be able to get the soft outputs concerning the information bits. Let $\mathcal{B}^{+}$be the set of state pairs $\left(\sigma^{\prime}, \sigma\right)$ such that the first info bit $b_{n}^{0}$ at time $n$ is +1 . Similarly define $\mathcal{B}^{-}$. The LLR value of the first information bit $b_{n}^{0}$ at the output of the TCM decoder is given by

$$
\begin{aligned}
\lambda\left(b_{n}^{0}\right)= & \max _{\mathcal{B}^{+}}\left[\alpha_{n}\left(\sigma^{\prime}\right)+\gamma_{n}\left(\sigma^{\prime}, \sigma\right)+\beta_{n+1}(\sigma)\right] \\
& -\max _{\mathcal{B}^{-}}\left[\alpha_{n}\left(\sigma^{\prime}\right)+\gamma_{n}\left(\sigma^{\prime}, \sigma\right)+\beta_{n+1}(\sigma)\right] .
\end{aligned}
$$

The soft value of the other information bits corresponding to the TCM symbol $s_{n}$ can be computed in a similar way. The branch metrics (probabilities) between states $\sigma^{\prime}$ and $\sigma$ are defined as

$$
\begin{aligned}
& \gamma_{n}\left(\sigma^{\prime}, \sigma\right)=\log p\left(y_{n}, \sigma \mid \sigma^{\prime}\right)=\log p\left(y_{n} \mid s_{n}\right)+\log P\left(s_{n}\right) \\
& \gamma_{n}^{*}\left(\sigma^{\prime}, \sigma\right)=\log p\left(y_{n} \mid s_{n}\right) .
\end{aligned}
$$

According to (10)

$$
\begin{aligned}
& y_{n}=\hat{\mathbf{h}}^{*} \mathbf{h} s_{n}+\eta \approx\|\mathbf{h}\|^{2} s_{n}+\eta=\mathcal{P} s_{n}+\eta \\
& p\left(y_{n} \mid s_{n}=s_{i}\right) \approx \frac{1}{\pi N_{\eta}} \exp \left(-\frac{\left|y_{n}-\mathcal{P} s_{i}\right|^{2}}{N_{\eta}}\right) \\
& =\frac{1}{\pi \mathcal{P} N_{v}} \exp \left(-\frac{\left|y_{n}\right|^{2}+\mathcal{P}^{2}\left|s_{i}\right|^{2}-2 \mathcal{P} \operatorname{Re}\left\{s_{i}^{*} y_{n}\right\}}{\mathcal{P} N_{v}}\right) .
\end{aligned}
$$

Omitting the common terms, e.g., $\frac{1}{\pi \mathcal{P} N_{v}} \exp \left(-\frac{\left|y_{n}\right|^{2}}{\mathcal{P} N_{v}}\right)$, which will be canceled in the subsequent calculations, we can denote

$$
p\left(y_{n} \mid s_{n}=s_{i}\right) \propto \exp \left(\frac{2 \operatorname{Re}\left\{s_{i}^{*} y_{n}\right\}-\mathcal{P}\left|s_{i}\right|^{2}}{N_{v}}\right) .
$$

Substituting (14) into (13) yields

$$
\begin{aligned}
& \gamma_{n}\left(\sigma^{\prime}, \sigma\right)=\frac{2 \operatorname{Re}\left\{s_{i}^{*} y_{n}\right\}-\mathcal{P}\left|s_{i}\right|^{2}}{N_{v}}+\log P\left(s_{n}=s_{i}\right), \\
& \gamma_{n}^{*}\left(\sigma^{\prime}, \sigma\right)=\frac{2 \operatorname{Re}\left\{s_{i}^{*} y_{n}\right\}-\mathcal{P}\left|s_{i}\right|^{2}}{N_{v}} .
\end{aligned}
$$

In (14) and (15), the term $\mathcal{P}\left|s_{i}\right|^{2}$ can be omitted for PSK signals since all the signal alternatives have the same energy. Note that the a priori probability $P\left(s_{n}\right)$ is dropped from $\gamma_{n}^{*}\left(\sigma^{\prime}, \sigma\right)$ to avoid statistic dependencies between the soft values of several iteration steps, so that only the extrinsic probabilities are fed back to the equalizer. With the LogMAP algorithm, $\alpha_{n}\left(\sigma^{\prime}\right)$ is computed recursively as $\alpha_{n}(\sigma)=$ $\max _{\sigma^{\prime}} *\left[\alpha_{n-1}\left(\sigma^{\prime}\right)+\gamma_{n}\left(\sigma^{\prime}, \sigma\right)\right]$, with initial conditions $\alpha_{0}(0)=$ $0, \alpha_{0}(\sigma \neq 0)=-\infty$. The max* operation is performed over all the states $\sigma^{\prime}$ where the transition $\left(\sigma^{\prime}, \sigma\right)$ is possible. The state metrics for the portion of the trellis beyond the $n^{\text {th }}$ node can be computed similarly by a backward recursion starting at the last node $\beta_{n-1}\left(\sigma^{\prime}\right)=\max _{\sigma}{ }^{*}\left[\beta_{n}(\sigma)+\gamma_{n}\left(\sigma^{\prime}, \sigma\right)\right]$, with initial conditions $\beta_{L_{b}}(0)=0, \beta_{L_{b}}(\sigma \neq 0)=-\infty$. The $\max ^{*}$ operation is performed over all the states $\sigma$ where the transition $\left(\sigma^{\prime}, \sigma\right)$ is possible. 


\begin{tabular}{|c|c|c|c|c|}
\hline \hline operations & multiplication/division & addition/subtraction & log/exp & max $^{*}$ \\
\hline Proposed scheme & $10 L^{2}+24$ & $L^{2}+8 L-2$ & 16 & 7 \\
\hline MMSE scheme & $8 L^{3}+4 L+39$ & $8 L^{3}-5 L^{2}+3 L+5$ & 8 & 7 \\
\hline
\end{tabular}

\section{NumericAl Results}

In this section, we present some simulation results to demonstrate the performance of the proposed scheme. In our experiments, we employ Ungerboeck's 8 -state 2/3 8PSK TCM [14]. In such a case, each pair of information bits $b_{n}^{0}, b_{n}^{1}$ at time instant $n$ correspond to one of $M=8$ PSK symbols $s_{n}$. During each Monte-Carlo run, 4096 coded symbols are interleaved by a $64 \times 64$ block interleaver and transmitted over an ISI channel. In order to ensure the reliability of the performance measurements, $100 \sim 500$ blocks of data are transmitted and at least 200 errors are generated. For the initial equalization, we use a DFE equalizer with 9 feedforward, 3 feedback taps. It uses 200 pilot symbols for training the equalizer coefficients (using RLS adaptation). In the meantime, the modified maximum likelihood algorithm introduced in [11] is used for channel estimation during the training period. For the computation of the branch metrics in the TCM decoding as expressed in (15), we found that in order to avoid numerical instability, it is necessary to do truncations, such as

$$
P\left(s_{n}=s_{i}\right)= \begin{cases}10^{-300} & \text { if } P\left(s_{n}=s_{i}\right)<10^{-300} \\ P\left(s_{n}=s_{i}\right) & \text { otherwise. }\end{cases}
$$

We observed from the experiments that replacing $N_{v}$, derived statistically with equation (4) with $N_{v}=N_{0}$ (assuming perfect cancellation), yields similar performance at low SNR and slightly better performance at high SNR, especially when the system reaches convergence. The reason is that $N_{v}$ calculated by (4) and assuming $N_{v}=N_{0}$ are both approximations; wheareas the latter will approach optimality when the ISI is effectively canceled as the iterative process proceeds. Therefore, perfect interference cancellation is assumed in our simulations.

The performance of the proposed scheme and the MMSE scheme is compared in Figure 3, where we use a 5-tap channel with impulse response $h[n]=(2-0.4 j) \delta[n]+(1.5+1.8 j) \delta[n-$ $1]+\delta[n-2]+(1.2-1.3 j) \delta[n-3]+(0.8+1.6 j) \delta[n-4]$, which is taken from [6]. The output channel power is normalized so that $P=\sum_{n=0}^{4}|h[n]|^{2}=1$. Results are shown for the $4^{\text {th }}$ stage at which all the schemes have converged. Upon convergence, the proposed scheme outperforms the original MMSE filterbased turbo equalization by $1 \mathrm{~dB}$ at a $\mathrm{BER}=10^{-4}$. In addition to the original scheme introduced in [6], [7], we also tested its improved version where the MMSE filter output is passed as the refined channel value to the TCM decoder in addition to the symbol a posteriori probabilities. Clearly, the improved algorithm performs better than the original one, meaning that it is necessary for the TCM decoder to be provided with the refined channel value in order to improve its decoding performance. The plot also shows that the proposed algorithm yields identical performance at low SNR and slightly superior

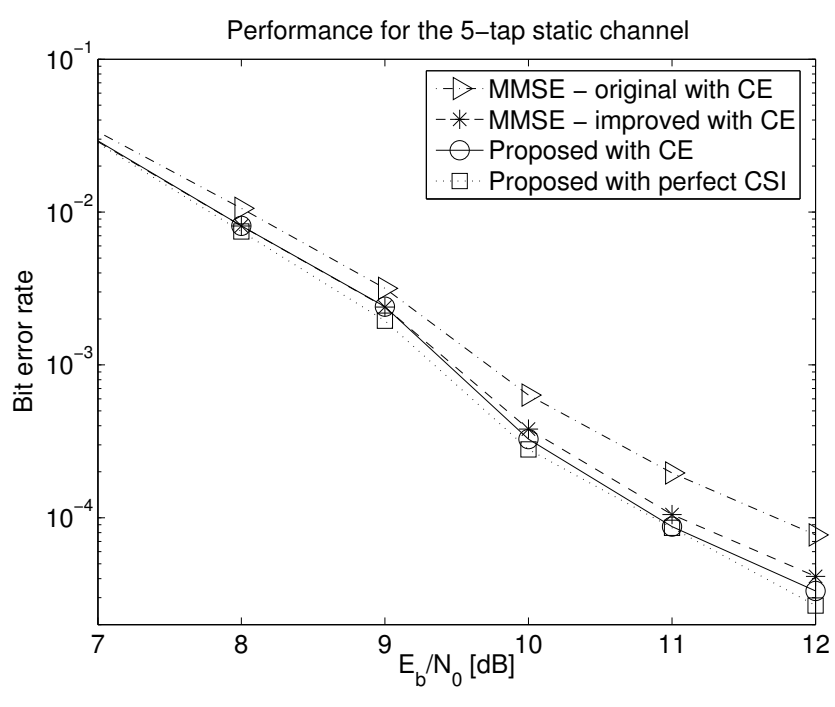

Fig. 3. Comparison between the proposed and the MMSE filter-based turbo equalization for the 5-tap channel. All the curves represent the $4^{t h}$ stage turbo equalization.

performance at high SNR to the improved version of the MMSE filter-based turbo equalization. However, the difference is not significant. Like the proposed scheme, the MMSE scheme is also suboptimum compared to the trellis based approach. The results show that the assumption of perfect cancellation does not incur a performance penalty compared to the MMSE scheme when the ISI is effectively canceled as the iterative process proceeds and the algorithm converges. Note that the approach proposed in Section II reduces the complexity from $O\left(L^{3}\right)$ to $O\left(L^{2}\right)$ by avoiding MMSE filtering and matrix inversion in the equalization process. The performance of the proposed turbo equalizer assuming perfect channel state information (CSI) is also shown in Figure 3. By comparison, we see that the performance loss due to imperfect channel estimation (CE) is very small compared to the genie-aided case assuming perfect CSI.

Figure 4 shows the performance of the proposed turbo equalizer for the 5-tap channel. It takes only 3 or 4 stages for the algorithm to converge. The gain achieved by iterative equalization and decoding is dramatic compared to separate equalization and decoding shown by the topmost curve.

Fig. 5 shows the performance comparison of different schemes in another static channel (Proakis B channel) having impulse response $h[n]=0.407 \delta[n]+0.815 \delta[n-1]+$ $0.407 \delta[n-2]$. Unlike the previous static channels which have a strong line-of-sight component (i.e., the first tap is stronger than the other taps), it is a much harsher channel and introduces much more frequency-selectivity. As indicated by 
Convergence of the proposed turbo equalizer

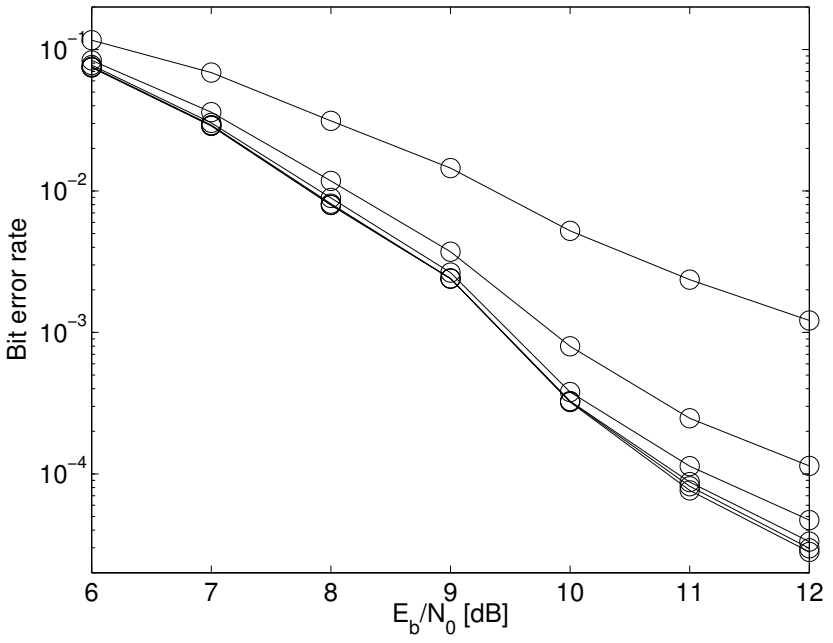

Fig. 4. Performance of the proposed turbo equalizer for the 5-tap channel. The topmost curve represents the first stage and the bottommost curve represents the $5^{\text {th }}$ stage.

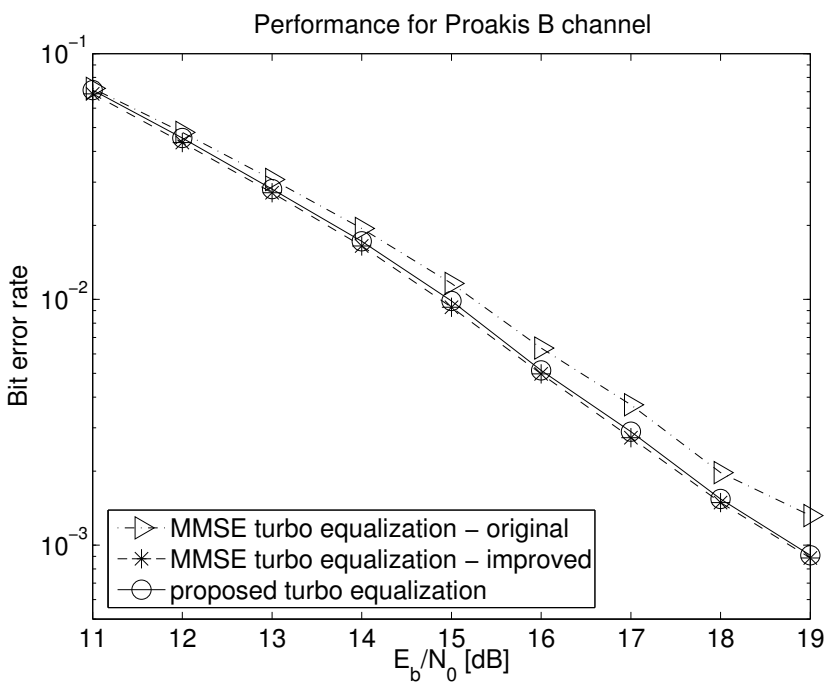

Fig. 5. Comparison between the proposed and the MMSE schemes for the Proakis B channel. All the curves represent the $4^{t h}$ stage turbo equalization.

Fig. 5, the improved version of the MMSE scheme has slightly better performance than the proposed scheme. However, the gap is very small. The gain obtained by performing MMSE filtering is negligible. Comparison between the two versions of the MMSE schemes shows that the improved scheme with refined channel value outperforms the original one.

\section{CONCLusions}

A new approach to iterative equalization and TCM decoding is investigated in this correspondence. Instead of only processing symbol a posteriori probabilities at TCM decoder as in the existing turbo equalization schemes, we have shown that the quality of decoding can be improved by passing the refined channel value from the equalizer to the TCM decoder. Simulation results of an 8-PSK TCM system show that the proposed scheme has superior (comparable) performance to the original (improved version of) MMSE filter-based turbo equalization. We believe that this is due to the nature of TCM, e.g., combined modulation and coding. No gain has been observed when a similar procedure is applied to a conventional system with independent modulation and coding (non-TCM system). In addition, the proposed equalizer has an affordable complexity, which is quadratic with the number of channel taps. It can operate on systems with high-order modulation and over channels with large delay spread, which is not feasible with the trellis-based approach.

\section{REFERENCES}

[1] C. Douillard, M. Jezequel, C. Berrou. "Iterative correction of intersymbol interference: turbo-equalization". European Transactions on Telecommunications, pp. 507-511, Sept. 1995.

[2] X. Wang, H. Poor. "Iterative (turbo) soft interference cancellation and decoding for coded CDMA". IEEE Transactions on Communications, vol. 47, no. 7, pp. 1046-1061, July 1999.

[3] M. Tuchler, R. Koetter, A. Singer. "Turbo equalization: principles and new results". IEEE Transactions on Communications, vol. 50, pp. 754 767, May 2002.

[4] M. Koca, B. Levy. "Turbo space-time equalization of TCM with receiver diversity I: symbol-by-symbol detection". In Proc. The Asilomar Conference on Signals, Systems and Computers, vol. 1, pp. 552-556, Oct. 2000.

[5] N. Doan, R. Rajatheva. "Turbo equalization for non-binary coded modulation schemes over frequency selective fading channels". In Proc. IEEE Vehicular Technology Conference, vol. 3, pp. 2217-2221, May 2000.

[6] A. Dejonghe, L. Vandendorpe. "Turbo-equalization for multilevel modulations: an efficient low-complexity scheme". In Proc. IEEE International Conference on Communications, vol. 3, pp. 1863-1867, April - May 2002.

[7] M. Koca, B. Levy. "Broadband beamforming for joint interference cancellation and low-complexity turbo equalization". In Proc. IEEE Global Telecommunications Conference, vol. 4, pp. 2161-2166, Dec. 2003.

[8] J. Proakis. Digital Communications, 4th edition, McGraw-Hill, 2000.

[9] M. Tuchler, A. Singer, R. Koetter. "Minimum mean square error equalization using a priori information". IEEE Transactions on Signal Processing, vol. 50, no. 3, pp. 673-683, March 2002.

[10] C. Laot, R. Bidan, D. Leroux. "Low-complexity MMSE turbo equalization: a possible solution to EDGE". IEEE Transactions on Wireless Communications, vol. 4, no. 3, pp. 965-974, May 2005.

[11] P. Xiao, R. Carrasco, I. Wassell. "Estimation of FWA MIMO channels". Proceedings IEEE Information Theory Workshop, ITW'2006, pp. 641645, Oct. 2006.

[12] P. Robertson, E. Villebrun, P. Hoeher. "A comparison of optimal and sub-optimal MAP decoding algorithms operating in the log domain". Proc. IEEE International Conference on Communications, pp. 10091013, 1995.

[13] P. Robertson, T. Worz. "Bandwidth-efficient turbo trellis-coded modulation using punctured component codes". IEEE Selected Areas in Communications, vol. 16, no. 2, Feb 1998.

[14] G. Ungerboeck. "Channel coding with multilevel/phase signals". IEEE Transactions on Information Theory, vol. 28, pp. 56-67, Jan. 1982. 